\title{
Force Vibration of Laminated Plates with Various Shapes Subjected to Low-velocity Impact
}

\author{
P. Yousefi ${ }^{1}$, Sh. Hosseini-Hashemi ${ }^{2}$ \& M. H. Kargarnovin ${ }^{3}$ \\ ${ }^{1}$ Mechanical Engineering Department, Science and Research Branch, Islamic Azad University (IAU), Tehran, \\ Iran \\ ${ }^{2}$ Mechanical Engineering Department, Iran University of Science and Technology, Tehran, Iran \\ ${ }^{3}$ Mechanical Engineering Department, Sharif University of Technology, Tehran, Iran \\ Correspondence: Peiman Yousefi, Mechanical Engineering Department, Science and Research Branch, Islamic \\ Azad University (IAU), Tehran, Iran. Tel: 98-861-313-2394. E-mail: y747y@yahoo.com
}

Received: May 3, $2012 \quad$ Accepted: May 17, $2012 \quad$ Online Published: June 12, 2012
doi:10.5539/jmsr.v1n3p106
URL: http://dx.doi.org/10.5539/jmsr.vln3p106

\begin{abstract}
In this paper, force vibration of composite laminate plates subjected to low-velocity impacts are investigated semi-analytically and analytically. Symmetrically angle-ply laminated rectangular, circular and elliptical plates are considered. The simply supported edges boundary condition is considered. Plates were studied based on small deflection thin plate theory. A Hertz Contact Theory models the contact forces between the rigid projectile and the laminated plate.

A simple and improved model was developed to calculate force vibration of composite plates. A semi-analytical model was obtained for force vibration of composite laminated plates by the method of Rayleigh-Ritz and modal expansion. Calculations of the impact response in composite material structures are important in damage-tolerant design. The results were compared with the results from the available literature and found to be in good agreement.
\end{abstract}

Keywords: force vibration, laminate plate, Hertz Contact Theory, Rayleigh-Rirz Method, modal expansion

\section{Introduction}

Composites are used in civil, aerospace applications, high-speed boats, sports supplies, automotive and transportation industries, where they are subjected to different type of loading. Their response to such conditions should be clearly understood. For instance, a tool can be dropped onto a composite during maintenance, or a flying fragment with low velocity can impact the composite structure. As a simple model, composite plates are impacted by spherical rigid impactors.

Different studies have been conducted to determine the dynamic properties of laminated plates. In a number of these studies, the closed form exact solutions have been presented for the natural frequencies of specially orthotropic, anti-symmetric angle-ply and anti-symmetric cross-ply laminated plates with simply-supported edges (Reddy, 1945; Whitney, 1987). Goldsmith (1960) organized and skillfully written text takes readers through the mathematical complexities of impact theory, after a brief introduction to the nature of physical impact, the text examines stereo-mechanical impact, vibrational aspects of impact, contact phenomena produced by impact of elastic bodies, dynamic processes involving plastic strains and result of impact experiments.

One of the most popular methods to obtain approximate solutions for the frequencies of an orthotropic plate is the Rayleigh-Ritz method. Nallim and Grossi (2008) presented the free transverse vibration analysis of symmetrically laminated solid and annular elliptic and circular plates based on the Rayleigh-Ritz method, where the deflection of the plate is approximated by a general shape function of a polynomial type. Lam and Chun (1994) have determined the dynamic response of laminated angle ply plates with the clamped boundary conditions subjected to explosive blast loading.

Shivakumar et al. (1985) developed two simple and improved models, energy-balance and spring-mass to calculate impact force and duration during low-velocity impact of circular composite plates. Aslan et al. (2003) concerned with evaluation of the in-plane dimensional effect of fiber-reinforced laminated composites under 
Low-velocity impact. A numerical simulation was performed using 3DIMPACT transient dynamic finite element analysis code for calculating stresses and contact forces of the composite plates during impact.

Sun and Chen (1985) used the three-dimensional finite element method to perform the dynamic analysis of laminated plates under impact loading. Cairns and Lagace (1989) considered influencing of different parameters on the impact behavior of laminated composite plates. A Rayleigh-Ritz energy method was used to spatially discretize the time-varying boundary value problem and a set of coupled ordinary differential equations in time were obtained based on the discretized system Lagrangian.

Chun and Kassegne (2005) used the higher-order shear deformation theory to study the response of graphite/epoxy laminated composite nonprismatic folded plates subjected to impact loads. A finite-element model of the theory is also developed. The modified Hertzian contact law incorporated within the Newton-Raphson method is used to calculate the contact force between the impactor and the laminated plate. Tiberkak et al. (2008) also investigated fiber-reinforced composite plates subjected to low velocity impact, by the use of finite element analysis. Dynamic stress analysis is carried out by the use of a constitutive equation of composite laminates without damage. And Setoodeh et al. (2009) analyzed a three-dimensional elasticity based approach coupled with layer-wise laminated plate theory employed to conduct low velocity impact analysis of general fiber reinforced laminated composite plates, with a finite element computation algorithm.

It is possible to theoretically state that there are no closed-form solutions for the calculation of frequencies of complicate shapes. The present study proposes a general, simple programming method for determining the natural frequency and mode shape of laminated angle-ply plates with various shapes by rectangular orthotropy. The relationship between the contact deformation and the contact force is generally modeled using Hertz contact laws. The effect of boundary shapes on the contact force of plates is considered. The objective of this paper, therefore, is to develop improved, yet simple, analyses to calculate the impact force and duration for low-velocity impact on laminated plates.

\section{Method}

\subsection{Free Vibration Analysis}

Consider a plate of total thickness $h$, composited of $n l$ (number of layer) orthotropic layers. The origin of the Cartesian coordinate system is located in the mid-plane with the $z$ axis perpendicular to the plane as shown in Figure 1. Let $u, v, w$ denote the mid-plane displacements in the directions of $x, y$ and $z$, respectively.
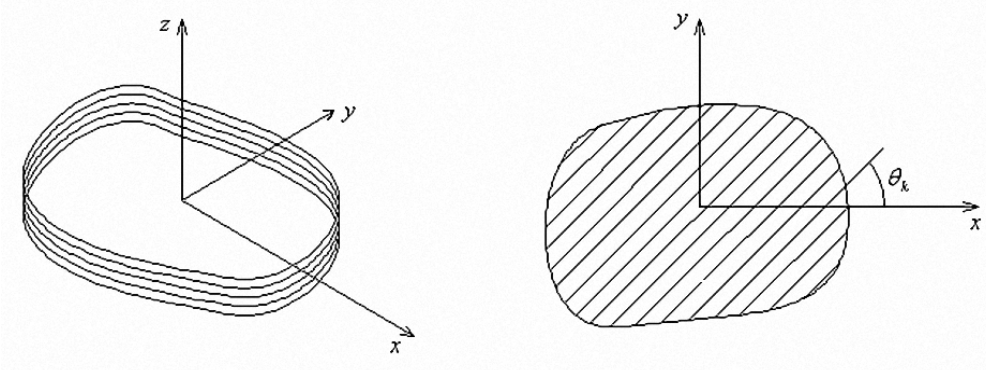

Figure 1. Geometry of the laminated plate and $k^{\text {th }}$ layer with the angle of fiber orientation $\theta_{k}$

The strain-displacement relations for the plate are (Reddy, 1945; Whitney, 1987)

$$
\varepsilon_{x}=\frac{\partial u}{\partial x}+z \kappa_{x} \quad \varepsilon_{y}=\frac{\partial v}{\partial y}+z \kappa_{y} \quad \varepsilon_{x y}=\frac{\partial u}{\partial y}+\frac{\partial v}{\partial x}+z \kappa_{x y}
$$

Where

$$
\kappa_{x}=-\frac{\partial^{2} w}{\partial x^{2}} \quad \kappa_{y}=-\frac{\partial^{2} w}{\partial y^{2}} \quad \kappa_{x y}=-2 \frac{\partial^{2} w}{\partial x \partial y}
$$

According to the generalized Hooke's law, the stress- strain relation for the $k^{\text {th }}$ layer is 


$$
\left[\begin{array}{c}
\sigma_{x} \\
\sigma_{y} \\
\sigma_{x y}
\end{array}\right]_{k}=[\bar{Q}]^{k}\left[\begin{array}{c}
\varepsilon_{x} \\
\varepsilon_{y} \\
\varepsilon_{x y}
\end{array}\right]_{k}
$$

Where

$$
\begin{gathered}
{[\bar{Q}]^{k}=[T]^{T}[Q]^{k}[T]} \\
{[T]=\left[\begin{array}{ccc}
\cos ^{2} \theta_{k} & \sin ^{2} \theta_{k} & \cos \theta_{k} \sin \theta_{k} \\
\sin ^{2} \theta_{k} & \cos ^{2} \theta_{k} & -\cos \theta_{k} \sin \theta_{k} \\
-2 \cos \theta_{k} \sin \theta_{k} & 2 \cos \theta_{k} \sin \theta_{k} & \cos ^{2} \theta_{k}-\sin ^{2} \theta_{k}
\end{array}\right]}
\end{gathered}
$$

In which $\theta_{k}$ is the angle of the fiber direction on the $k^{t h}$ layer with the $x$-axis, as shown in Figure $1 .[Q]^{k}$ is the stiffness matrix of the $k^{\text {th }}$ layer and its components are given by:

$$
\begin{aligned}
& Q_{11}=\frac{E_{11}}{1-v_{12} v_{21}} \quad Q_{22}=\frac{E_{22}}{1-v_{12} v_{21}} \quad Q_{12}=Q_{21}=\frac{v_{12} E_{22}}{1-v_{12} v_{21}}=\frac{v_{21} E_{11}}{1-v_{12} v_{21}} \\
& Q_{66}=G_{12} \quad Q_{16}=Q_{26}=0
\end{aligned}
$$

Where $E_{11}$ and $E_{22}$ are the Young's modulus, $G_{12}$ is the shear modulus and $v_{12}$ are the Poisson's ratios. It is unlikely that an exact solution can be obtained for various shapes and boundary conditions. The Rayleigh-Ritz method was employed to obtain a semi-analytical solution. A set of simple polynomials is selected as the admissible function for plate displacement. The plate is assumed to be in harmonic motion and hence the displacements can be written as:

$$
\begin{array}{r}
u(x, y, t)=U(x, y) \cos (\omega t) \\
v(x, y, t)=V(x, y) \cos (\omega t) \\
w(x, y, t)=W(x, y) \cos (\omega t)
\end{array}
$$

Where $\omega$ natural frequency and $U, V, W$ can be denoted in terms of $x$ and $y$ (Yousefi et al. 2011)

$$
\begin{gathered}
\left.U(x, y)=(\text { Boundary }- \text { shape }- \text { Function }) \sum_{i=0}^{p} \sum_{j=0}^{i} \alpha_{\left(\frac{(i+1)(i+2)}{2}-j\right.}\right)^{x^{j}} y^{i-j} \\
\left.V(x, y)=(\text { Boundary }- \text { shape }- \text { Function }) \sum_{i=0}^{p} \sum_{j=0}^{i} \alpha_{\left(n t+\frac{(i+1)(i+2)}{2}-j\right.}\right)^{x^{j} y^{i-j}} \\
\left.W(x, y)=(\text { Boundary }- \text { shape }- \text { Function })^{n} \sum_{i=0}^{p} \sum_{j=0}^{i} \alpha_{\left(2 n t+\frac{(i+1)(i+2)}{2}-j\right.}\right)^{x^{j} y^{i-j}}
\end{gathered}
$$

For Boundary-shape-function see Table 1 and $n=1,2$ satisfied geometry boundary condition of plate, $n=1$ for simple $(w=0)$ and $n=2$ for $\operatorname{clamped}(w=0, \partial w / \partial n=0)$. Also $p$ is maximum power of polynomial, $\alpha_{1}, \alpha_{2}, \ldots$ are the arbitrary coefficients and $n t=\frac{(p+1)(p+2)}{2}$ (number of polynomial terms). 
Table 1 . Shapes and boundary shapes function

Shape
Boundary shape function
Rectangular

From the elastic theory, the strain and kinetic energies are written as:

$$
U_{\text {eng }}=\frac{1}{2} \sum_{k=1}^{n l} \int_{V_{k}}\left(\sigma_{x} \varepsilon_{x}+\sigma_{y} \varepsilon_{y}+\sigma_{x y} \varepsilon_{x y}\right) d V \quad T_{\text {eng }}=\frac{1}{2} \sum_{k=1}^{n l} \int_{V_{k}} \rho_{x}\left(\dot{u}^{2}+\dot{v}^{2}+\dot{w}^{2}\right) d V
$$

Where $n l$ is the total number of layers and $V_{k}$ is the $k^{\text {th }}$ layer volume. Substituting Eqs. $(8,7,3,2,1)$ into Eq. 9 , the maximum strain and kinetic energies are obtained:

$$
U_{\max }=\frac{1}{2}[X]^{T}[K][X] \quad T_{\max }=\frac{1}{2} \omega^{2}[X]^{T}[M][X]
$$

Where

$$
[X]^{T}=\left[\alpha_{1}, \alpha_{2}, \alpha_{3}, \cdots, \alpha_{3 n t}\right]
$$

While $[K]$ and $[M]$ are the $3 \times n t$ dimension, stiffness and mass matrixes. Using the Rayleigh-Ritz principle can be writing:

$$
\left(\frac{\partial}{\partial \alpha_{i}}\right)\left(T_{\max }-U_{\max }\right)=0
$$

Results are a set of homogeneous equations in an equal number of unknown $\alpha_{i}$. These equations can be described in matrix form:

$$
\left([K]-\omega^{2}[M]\right)[X]=0
$$

This will yield a set of frequencies by determine the Eigen-values $(\lambda)$ of $[M]^{-1}[K]$ as shown in Table 2 $\left(\omega^{2}=\lambda\right)$. The corresponding mode shapes can be obtained by Eigen-vectors of $[M]^{-1}[K]$ as shown in Table 3 . It is a fact that fundamental frequencies obtained using the semi-analytical Rayleigh-Ritz method are always higher than the exact values since the plate mode shape assumed by a finite number of terms in the shape functions which inherently increase the rigidity of the plate. An analytical method is available for a narrow range of condition. The method is restricted to simply supported boundary conditions, and especially orthotropic laminated rectangular plates. In this case the transverse displacement $w$ same as isotropic plate is

$$
w(x, y, t)=\sum_{n=1}^{\infty} \sum_{m=1}^{\infty} \sin \left(\frac{n \pi x}{a}\right) \sin \left(\frac{m \pi y}{b}\right) \cos \left(\omega_{n m} t\right)
$$

And set of natural frequencies determine as:

$$
\omega_{n m}^{2}=\frac{\pi^{4}}{\rho h}\left[D_{11}\left(\frac{n}{a}\right)^{4}+2\left(D_{12}+2 D_{66}\right)\left(\frac{n}{a}\right)^{2}\left(\frac{m}{b}\right)^{2}+D_{22}\left(\frac{m}{b}\right)^{4}\right]
$$

This approach is particularly useful as comparison with above semi-analytical Rayleigh-Ritz method. 
Table 2. First four natural frequency $(\mathrm{rad} / \mathrm{sec})$ of laminated plate by presented analytical and semi-analytical method

\begin{tabular}{ccccccc}
\hline Boundary & \multirow{2}{*}{$\begin{array}{c}\text { Dimensions } \\
\text { Conditions }\end{array}$} & Method & \multicolumn{4}{c}{ Mode sequence number } \\
\cline { 4 - 7 } & & & 1 & 2 & 3 & 4 \\
\hline $\begin{array}{c}\text { Simply Supported } \\
\text { Rectangular Plate }\end{array}$ & $a=b=0.2$ & Analytical & 1900.3828 & 4699.1181 & 5806.4911 & 7601.5313 \\
$\begin{array}{c}\text { Simply Supported } \\
\text { Circular Plate }\end{array}$ & $R=0.1128$ & Present & 1713.3704 & 4365.0292 & 5245.4695 & 8165.4948 \\
$\begin{array}{c}\text { Simply Supported } \\
\text { Elliptical Plate }\end{array}$ & $a=0.0921, b=1.5 a$ & Present & 2105.9549 & 3794.5583 & 6655.8467 & 7301.0961 \\
$\begin{array}{c}\text { Simply Supported } \\
\text { Elliptical Plate }\end{array}$ & $a=0.0798, b=2 a$ & Present & 2613.3996 & 3951.5162 & 6058.5862 & 9001.9731 \\
\hline
\end{tabular}

Table 3. First four Mode-shape of laminated plate by presented semi-analytical method

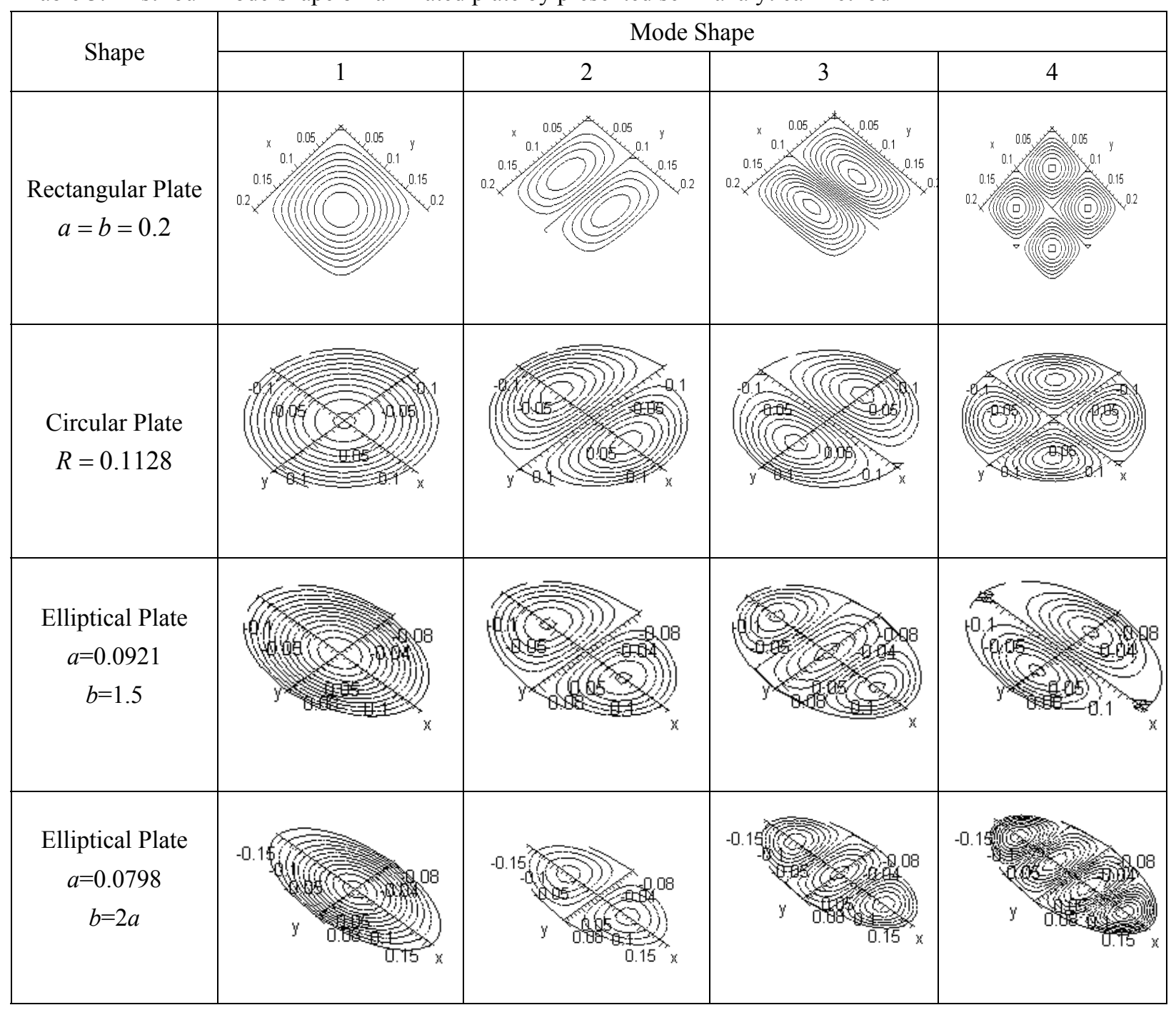

\subsection{Dynamic Response}

Plate is assumed to be subjected to a normal pressure $P(x, y, t)$ on the upper surface. The linear equations of motion of classical plate theory for laminates with constant A's, B's and D's are (Reddy, 1945). 


$$
\begin{gathered}
A_{11} \frac{\partial^{2} u}{\partial x^{2}}+2 A_{16} \frac{\partial^{2} u}{\partial x \partial y}+A_{66} \frac{\partial^{2} u}{\partial y^{2}}+A_{16} \frac{\partial^{2} v}{\partial x^{2}}+\left(A_{12}+A_{66}\right) \frac{\partial^{2} v}{\partial x \partial y}+A_{26} \frac{\partial^{2} v}{\partial y^{2}} \\
-B_{11} \frac{\partial^{3} w}{\partial x^{3}}-3 B_{16} \frac{\partial^{3} w}{\partial x^{2} \partial y}-\left(B_{12}+2 B_{66}\right) \frac{\partial^{3} w}{\partial x \partial y^{2}}-B_{26} \frac{\partial^{3} w}{\partial y^{3}}=\rho h \frac{\partial^{2} u}{\partial t^{2}} \\
A_{16} \frac{\partial^{2} u}{\partial x^{2}}+\left(A_{12}+A_{66}\right) \frac{\partial^{2} u}{\partial x \partial y}+A_{26} \frac{\partial^{2} u}{\partial y^{2}}+A_{66} \frac{\partial^{2} v}{\partial x^{2}}+2 A_{26} \frac{\partial^{2} v}{\partial x \partial y}+A_{22} \frac{\partial^{2} v}{\partial y^{2}} \\
-B_{16} \frac{\partial^{3} w}{\partial x^{3}}-\left(B_{12}+2 B_{66}\right) \frac{\partial^{3} w}{\partial x^{2} \partial y}-3 B_{26} \frac{\partial^{3} w}{\partial x \partial y^{2}}-B_{22} \frac{\partial^{3} w}{\partial y^{3}}=\rho h \frac{\partial^{2} v}{\partial t^{2}} \\
D_{11} \frac{\partial^{4} w}{\partial x^{4}}+4 D_{16} \frac{\partial^{4} w}{\partial x^{3} \partial y}+2\left(D_{12}+2 D_{66}\right) \frac{\partial^{4} w}{\partial x^{2} \partial y^{2}}+4 D_{26} \frac{\partial^{4} w}{\partial x \partial y^{3}}+D_{22} \frac{\partial^{4} w}{\partial y^{4}} \\
-B_{11} \frac{\partial^{3} u}{\partial x^{3}}-3 B_{16} \frac{\partial^{3} u}{\partial x^{2} \partial y}-\left(B_{12}+2 B_{66}\right) \frac{\partial^{3} u}{\partial x \partial y^{2}}-B_{26} \frac{\partial^{3} u}{\partial y^{3}}-B_{16} \frac{\partial^{3} v}{\partial x^{3}} \\
-\left(B_{12}+2 B_{66}\right) \frac{\partial^{3} v}{\partial x^{2} \partial y}-3 B_{26} \frac{\partial^{3} v}{\partial x \partial y^{2}}-B_{22} \frac{\partial^{3} v}{\partial y^{3}}+P(x, y, t)=\rho h \frac{\partial^{2} w}{\partial t^{2}}
\end{gathered}
$$

Where

$$
A_{i j}, B_{i j}, D_{i j}=\sum_{k=1}^{n l} \int_{z_{k}}^{z_{k+1}} \bar{Q}_{i j}^{k}\left(1, z, z^{2}\right) d z
$$

Assume modal expansion for transverse displacement

$$
\begin{gathered}
u=\sum_{n=1}^{\infty} \sum_{m=1}^{\infty} \widetilde{U}_{n m}(x, y) T_{n m}(t), \\
v=\sum_{n=1}^{\infty} \sum_{m=1}^{\infty} \widetilde{V}_{n m}(x, y) T_{n m}(t), \\
w=\sum_{n=1}^{\infty} \sum_{m=1}^{\infty} \widetilde{W}_{n m}(x, y) T_{n m}(t)
\end{gathered}
$$

Where $\widetilde{U}_{n m}(x, y), \widetilde{V}_{n m}(x, y), \widetilde{W}_{n m}(x, y)$ are the plate mode shapes. By substitution of Eq. 18 in the last Eq. 16 yields:

$$
\begin{aligned}
\sum_{n=1}^{\infty} \sum_{m=1}^{\infty}\left(D_{11} \widetilde{W}_{, x x x x}\right. & \left.+4 D_{16} \widetilde{W}_{, x x x y}+2\left(D_{12}+2 D_{66}\right) \widetilde{W}_{, x x y y}+\cdots\right) T_{n m}(t)+P(x, y, t) \\
& =\rho h \sum_{n=1}^{\infty} \sum_{m=1}^{\infty} \widetilde{W}_{n m} \ddot{T}_{n m}(t)
\end{aligned}
$$

Since the mode shapes satisfy the free vibration equation

$$
\begin{gathered}
\sum_{n=1}^{\infty} \sum_{m=1}^{\infty}\left(D_{11} \widetilde{W}_{, x x x x}+4 D_{16} \widetilde{W}_{, x x x y}+2\left(D_{12}+2 D_{66}\right) \widetilde{W}_{, x x y y}+\cdots\right) T_{n m}(t) \\
=-\rho h \sum_{n=1}^{\infty} \sum_{m=1}^{\infty} \widetilde{W}_{n m} \omega_{n m}^{2} T_{n m}(t)
\end{gathered}
$$

Substitution of Eq. 20 into Eq. 19 yields:

$$
-\rho h \sum_{n=1}^{\infty} \sum_{m=1}^{\infty} \widetilde{W}_{n m} \omega_{n m}^{2} T_{n m}(t)+P(x, y, t)=\rho h \sum_{n=1}^{\infty} \sum_{m=1}^{\infty} \widetilde{W}_{n m} \ddot{T}_{n m}(t)
$$


Multiplication Eq. 21 by $\widetilde{W}_{n^{\prime} m^{\prime}}$ and integration over the area of the plate.

$$
\ddot{T}_{n m}(t) \iint_{A} \rho h \widetilde{W}_{n m} \tilde{W}_{n^{\prime} m^{\prime}} d y d x+\omega_{n m}^{2} T_{n m}(t) \iint_{A} \rho h \widetilde{W}_{n m} \tilde{W}_{n^{\prime} m^{\prime}} d y d x=\iint_{A} P(x, y, t) \widetilde{W}_{n^{\prime} m^{\prime}} d y d x
$$

By the orthogonality of the mode shapes, $\iint_{A} \widetilde{W}_{n m} \widetilde{W}_{n^{\prime} m^{\prime}} d y d x\left\{\begin{array}{ll}=0 & n, m \neq n^{\prime}, m^{\prime} \\ \neq 0 & n, m=n^{\prime}, m^{\prime}\end{array}\right.$ and assuming $K_{n m}=\iint_{A} \rho h \widetilde{W}_{n m}^{2} d y d x \quad, \quad Q_{n m}=\iint_{A} P(x, y, t) \widetilde{W}_{n m} d y d x$, the following equation obtained:

$$
\ddot{T}_{n m}(t)+\omega_{n m}^{2} T_{n m}(t)=\frac{Q_{n m}(t)}{\rho h K_{n m}}
$$

The solution of Eq. 23 is obtained by using the convolution integral.

$$
T_{n m}(t)=\frac{1}{\rho h K_{n m} \omega_{n m}^{2}} \int_{0}^{t} Q_{n m}(\tau) \sin \left(\omega_{n m}(t-\tau)\right) d \tau
$$

By time depend point force $F(t)$ at $\left(x_{0}, y_{0}\right), P(x, y, t)=F(t) \delta\left(x-x_{0}\right) \delta\left(y-y_{0}\right)$

$$
T_{n m}(t)=\frac{\tilde{W}_{n m}\left(x_{0}, y_{0}\right)}{\rho h K_{n m} \omega_{n m}^{2}} \int_{0}^{t} F(\tau) \sin \left(\omega_{n m}(t-\tau)\right) d \tau
$$

Hence forced plate displacement is:

$$
w=\sum_{n=1}^{\infty} \sum_{m=1}^{\infty} \frac{\tilde{W}_{n m} \tilde{W}_{n m}\left(x_{0}, y_{0}\right)}{\rho h K_{n m} \omega_{n m}^{2}} \int_{0}^{t} F(\tau) \sin \left(\omega_{n m}(t-\tau)\right) d \tau
$$

In the semi-analytical model, the mode shapes can be obtained by substitute the Eigen-vectors of $[M]^{-1}[K]$ in Eq. 8 and in the analytical model, the mode shapes are

$$
W(x, y)=\sin \left(\frac{n \pi x}{a}\right) \sin \left(\frac{m \pi y}{b}\right)
$$

Contact law is the relationship between the contact force and the indentation, where the indentation is the difference between the displacement of the impactor tip and that of the top surface of the plate when the projectile has a higher rigidity than the plate. In Low-velocity impact, where the duration of impact is long in comparison to the period of the lowest vibration mode of the plate, the Hertz contact law can be applied. Hertzian theory can be modified to apply for the case of impact on an anisotropic surface like composite laminates. During loading, the contact force $F$ can be evaluated using the modified Hertz contact law as follows (Goldsmith, 1960):

$$
F(t)=k[\alpha(t)]^{3 / 2}
$$

Where $\alpha$ is the indentation, and $k$ is the modified Hertz contact stiffness between the composite plate and rigid foreign impactor, which is defined as:

$$
k=\frac{4 \sqrt{R_{s}}}{3\left[\left(1-v_{s}^{2}\right) / E_{s}+1 / E_{22}\right]} \approx \frac{4 E_{22}}{3} \sqrt{R_{s}}
$$

$E_{22}$ is the transverse Young's modulus of the composite plate, and $R_{s}, v_{s}, E_{s}$ are the radius, Poisson's ratio and Young's modulus of the spherical impactor.

The dynamic response of the impact is investigated with the consideration of these assumptions: 1. frictionless between the impactor and composite plate; 2. neglecting the damping effect in the composite plate; 3 . the impactor is a rigid body with isotropic properties; 4 . only the first impact is considered. 


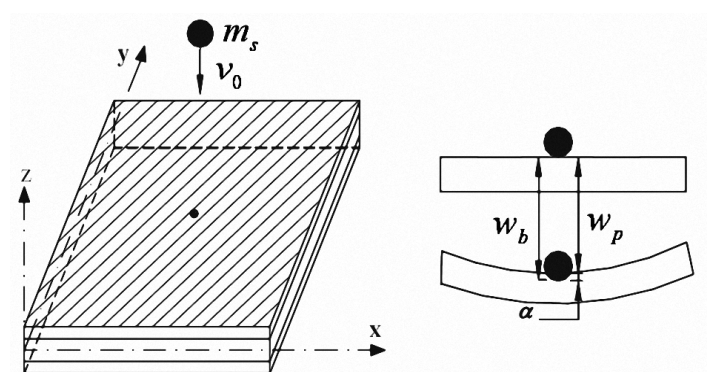

Figure 2. Laminated plate and impactor

As a simplifying approximation, the contact force $F$ is simply taken as a point force and by Hertz contact theory, the indentation $\alpha$ is defined as $\alpha(t)=w_{b}(t)-w_{p}(t), w_{b}(t)$ is the impactor displacement and $w_{p}(t)$ is the transverse plate displacement at the impact point, see Figure 2.

$$
\begin{aligned}
& w_{b}=v_{0} t-\frac{1}{m_{s}} \int_{0}^{t}\left(\int_{0}^{t} F(t) d t\right) d t, \\
& w_{p}=\frac{\widetilde{W}_{n m} \widetilde{W}_{n m}\left(x_{0}, y_{0}\right)}{\rho h K_{n m} \omega_{n m}^{2}} \int_{0}^{2} F(\tau) \sin \left(\omega_{n m}(t-\tau)\right) d \tau
\end{aligned}
$$

Hence:

$$
\begin{aligned}
\alpha(t) & =\left[\frac{F(t)}{k}\right]^{2 / 3} \\
& =v_{0} t-\frac{1}{m_{s}} \int_{0}^{t}\left(\int_{0}^{t} F(t) d t\right) d t-\sum_{n=1}^{\infty} \sum_{m=1}^{\infty} \frac{\widetilde{W}_{n m} \widetilde{W}_{n m}\left(x_{0}, y_{0}\right)}{\rho h K_{n m} \omega_{n m}^{2}} \int_{0}^{t} F(\tau) \sin \left(\omega_{n m}(t-\tau)\right) d \tau
\end{aligned}
$$

The solution of Eq. 31 with time increment gives the impact force in the impact time duration. First, the response of the plate to a unit impulse of time duration $\Delta t$ is calculated for the entire time history and stored in the memory. After that the non-linear Hertz law is applied at every time step $\left(\Delta t=\frac{1}{1000}\left(\frac{2 \pi}{\omega_{11}}\right)\right)$ to calculate the impact force. The impact force at any time $t$ is calculated from the impactor and plate displacements of the previous time step. Each term of above equation extract in Appendix.

\section{Results}

In this section examples of composite plate are investigated the achieved results of solving the problem with the use of the present theory are compared to the available results to assure validity. Four shapes simply-supported ten layered carbon fiber reinforced plate with $(0 / 90 / 0 / 90 / 0)$ s stacking sequences were considered. The material properties are assumed equal for all layers. The mechanical properties of each layer are:

$$
\begin{gathered}
E_{11}=120 \mathrm{GPa}, E_{22}=E_{33}=7.9 \mathrm{GPa}, G_{12}=G_{13}=G_{23}=5.5 \mathrm{GPa}, \\
v_{12}=v_{13}=v_{23}=0.3, \rho=1580 \mathrm{Kg} / \mathrm{m}^{3}
\end{gathered}
$$

These data are material properties of T300/934 taken from Sun and Chen (1985). The presented solution for plates is obtained by using MAPLE-10. For all composite plate shapes (square, circular and elliptical) by equal thickness and area: $h=2.69 \mathrm{~mm} a=b=200 \mathrm{~mm}$ for square plate, $R=112.8 \mathrm{~mm}$ for circular plate, $a=92.1 \mathrm{~mm}, b=1.5 a$ and $a=79.8 \mathrm{~mm}, b=2 a$ for elliptical plate.

\subsection{Free Vibration}

Table 2 gives first four natural-frequencies of composite plate with different shape (square, circular, elliptical), simply supported boundary condition and $(0 / 90 / 0 / 90 / 0)_{s}$ stacking sequences computed in the present study based on 136 terms of sires used in semi-analytically Rayleigh-Ritz method. And also gives a comparison of semi-analytical solution with analytical solution for especially orthotropic simply support laminated square plate. Excellent agreement is shown. First four mode-shapes of these four plates are presented in Table 3. 


\subsection{Force Vibration}

The force caused by the impact of a sphere on a composite plate cannot be expressed by a simple and known analytical function of time. Thus the force history profile is idealized by a suitable step curve having constant forces over equal time increments. The plate was impacted at the center by a spherical steel ball of $12.7 \mathrm{~mm}$ diameter with an initial velocity of $3 \mathrm{~m} / \mathrm{s}$. The mass density of the impactor is $7960 \mathrm{Kg} / \mathrm{m}^{3}$. The modified Hertzian contact stiffness assumed in the analysis is taken to be $k_{c}=8.394 \times 10^{8} \mathrm{~N} / \mathrm{m}^{1.5}$.

The time history of the impact process such as impactor displacement and the target fiber-reinforced composite plate deflection due to impact force acting at the center has been calculated. The effect of plate shape is examined. For impact behavior, validation of the presented analytically and semi-analytically method was carried out using the same example used by Sun and Chen (1985), Cairns and Lagace (1989) and Chun and Kassegne (2005). 136 mode-shapes of Rayleigh-Ritz method and 625 mode-shapes of analytical method are used for the analysis of folded plates subjected to impact loads. It may be seen in Figure 3 that obtained contact force history by analytical method is in good agreement with the results reported by previous investigators, but obtained contact peak force by semi-analytically method about $40 \%$ more than analytically. The results show the relatively large number of modes that are required to achieve good results.

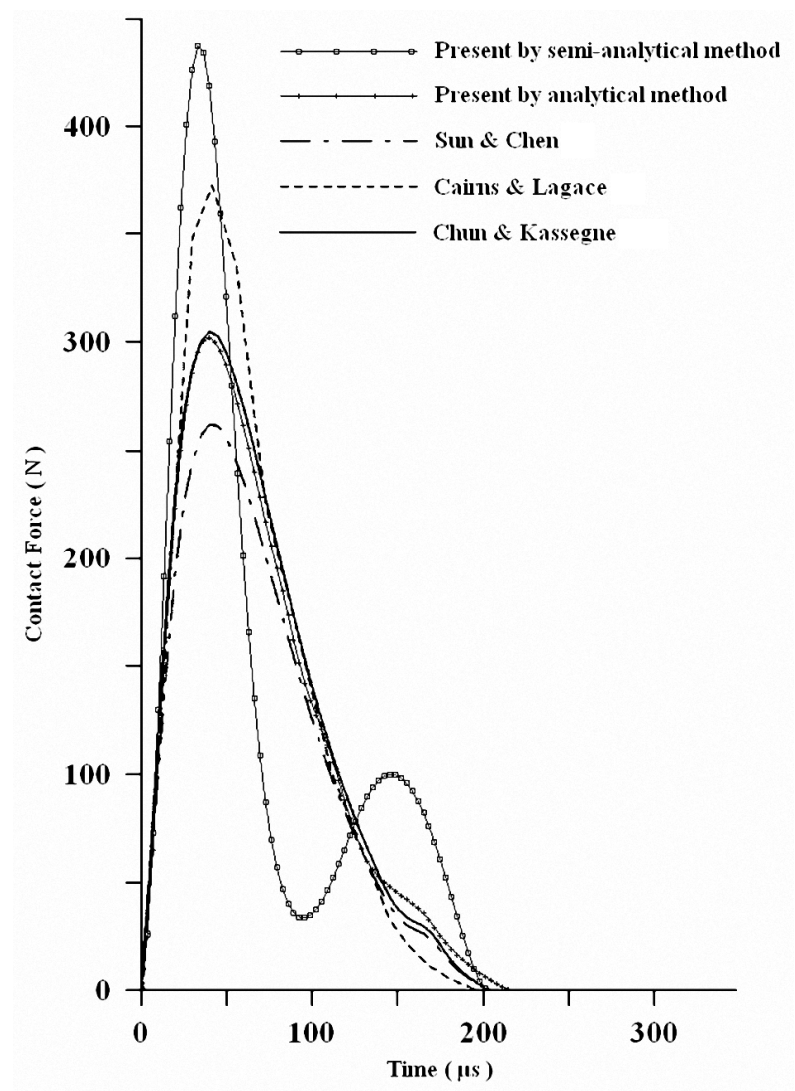

Figure 3. Contact force history

\section{Discussion}

The analysis led to the following conclusions:

(a) Impact force obtained from semi-analytically Rayleigh-Ritz method by 136 mode shapes was little greater than analytically method by $25 \times 25$ mode shapes, but semi-analytically method is simple, and approximately predicts the maximum impact force (Figure 3).

(b) Impact forces calculated from the two models by high number of mode-shape agreed with each other and with reported data (Figure 3).

(c) The contact force depends to plate shape. The calculated impact forces for rectangular plate are greater than elliptical and elliptical plate greater than circular (Figure 4). 
(d) Semi-analytically Rayleigh-Ritz method developed for the history of contact force, transverse plate deflection at contact point and projectile displacement, as shown in Figures 4, 5.

(e) Presented method can be used to study the responses of laminated plates with any stacking sequence, boundary conditions and plate shapes under different type of point loading.

(f) The impact peak force by approximate semi-analytically is higher than analytically and exact value. But it's able to find for a wide range of different plate shape having an internal hole, internal ring support, any aspect ratio and different boundary condition with simple programming. A presented study has been conducted prior to damage prediction.

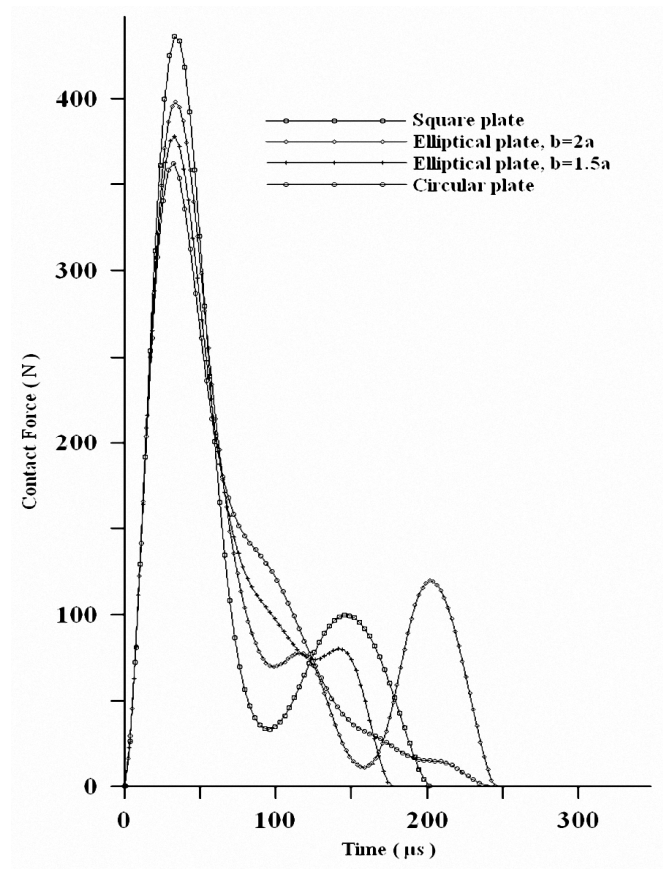

Figure 4. Contact force history of obtained plate shape by semi-analytical method
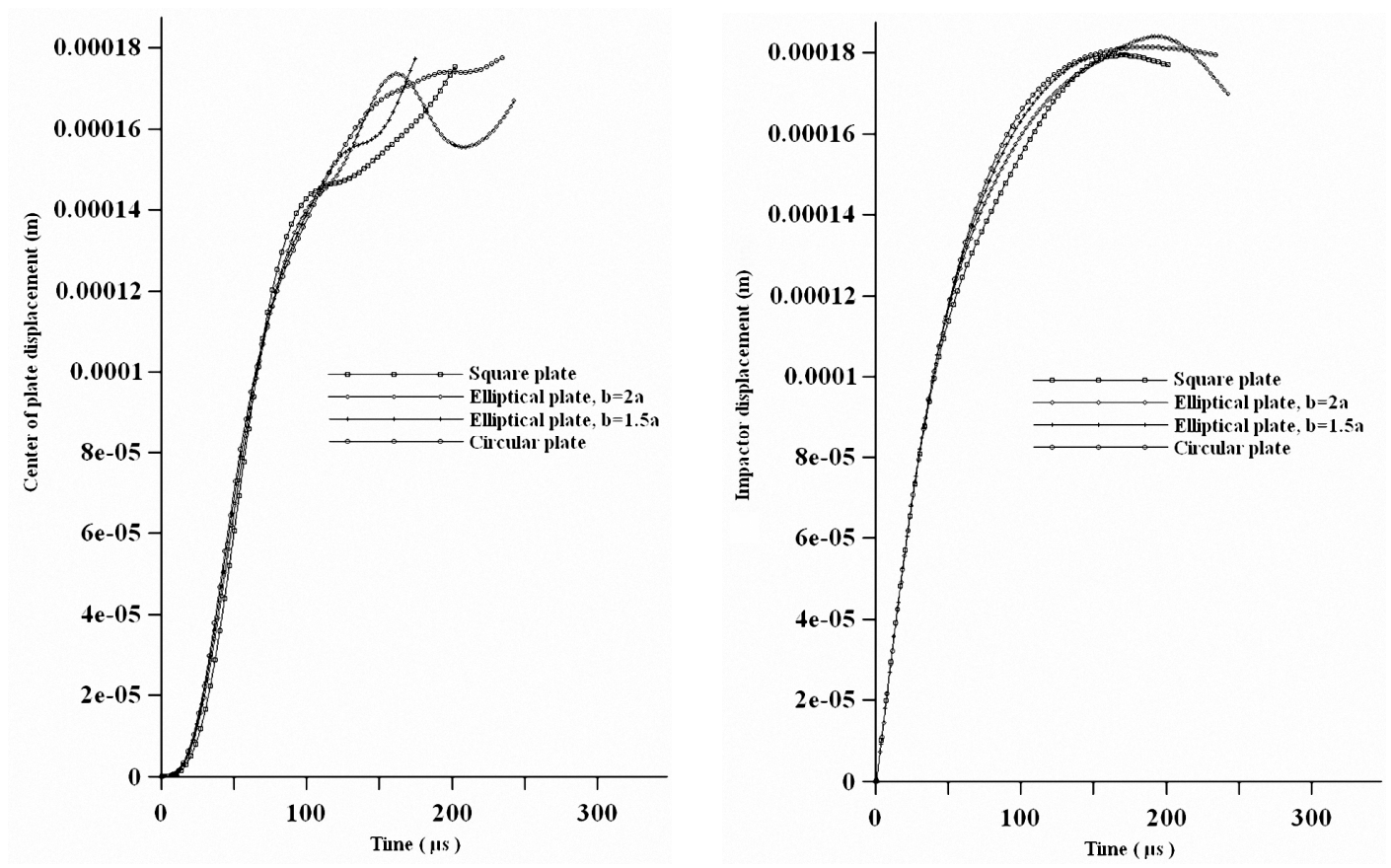

Figure 5. Center of plate and Impactor displacement in impact duration 


\section{References}

Aslan, Z., Karakuzu, R., \& Okutan, B. (2003). The response of laminated composite plates under low-velocity impact loading. Composite Structures, 59, 119-127. http://dx.doi.org/10.1016/S0263-8223(02)00185-X

Cairns, D. S., \& Lagace, P. A. (1989) .Transient response of graphite/epoxy and kevlar/epoxy laminates subjected to impact. AIAA Journal, 27(11), 1590-1596. http://dx.doi.org/10.2514/3.10306

Chun, K. S., \& Kassegne, S. K. (2005). Low-Velocity Impact Dynamic Behavior of Laminated Composite Nonprismatic Folded Plate Structures. Journal of Engineering Mechanics, 131(7), 678-688. http://dx.doi.org/10.1061/(ASCE)0733-9399(2005)131:7(678)

Goldsmith, W. (1960). Impact: the theory and physical behaviour of colliding solids. London: Edward Arnold.

Lam, K. Y., \& Chun, L. (1994). Analysis of clamped laminated plates subjected to conventional blast. Composite Structures, 29, 311-321. http://dx.doi.org/10.1016/0263-8223(94)90027-2

Nallim, L. G., \& Grossi, R. O. (2008). Natural frequencies of symmetrically laminated elliptical and circular

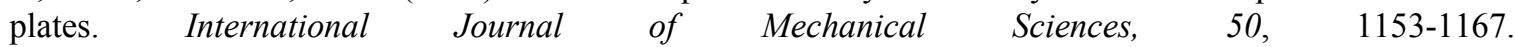
http://dx.doi.org/10.1016/j.jimecsci.2008.04.005

Reddy, J. N. (1945). Mechanics of laminated composite plates and shells: theory and analysis. Florida: CRC Press.

Setoodeh, A. R., Malekzadeh, P., \& Nikbin, K. (2009). Low-velocity impact analysis of laminated composite plates using a 3D elasticity based layer-wise FEM. Materials and Design, 30, 3795-3801. http://dx.doi.org/10.1016/j.matdes.2009.01.031

Shivakumar, K. N., Elber,W., \& Illg, W. (1985). Prediction of Impact Force and Duration Due to Low-Velocity Impact on Circular Composite Laminates. Journal of Applied Mechanics, 52, 674-680. http://dx.doi.org/10.1115/1.3169120

Sun, C. T., \& Chen, J. K. (1985). On the impact of initially stressed composite laminates. Journal of Comput Math, 19(11), 490-504.

Tiberkak, R., Bachene, M., Rechak, S., \& Necib, B. (2008). Damage prediction in composite plates subjected to low-velocity impact. Composite Structures, 83, 73-82. http://dx.doi.org/10.1016/j.compstruct.2007.03.007

Whitney, J. M. (1987). Structural analysis of laminated anisotropic plates. Lancaster, PA: Technomic.

Yousefi, P., Kargarnovin, M. H., \& Hosseini-Hashemi, S. H. (2011). Free Vibration of Generally Laminated Plates with Various Shapes. Polymer Composites, 32, 445-454. http://dx.doi.org/10.1002/pc.21063

\section{Appendix}

To fined impact force in the impact time duration by time increment $\Delta t$, we have:

$$
\begin{gathered}
v_{0} t \rightarrow v_{0} \times k \Delta t \\
\left.\frac{1}{m_{s}} \int_{0}^{t} \int_{0}^{t} F(t) d t\right) d t \rightarrow \sum_{i=1}^{k} \frac{1}{m_{s}} F_{i} \Delta t^{2}\left(k-i+\frac{1}{2}\right) \\
\int_{0}^{t} F(\tau) \sin \left(\omega_{n m}(t-\tau)\right) d \tau \rightarrow \\
\frac{1}{\omega_{n m}} \sum_{i=1}^{k} F_{i}\left\{\cos \left[\omega_{n m}((k-i) \Delta t)\right]-\cos \left[\omega_{n m}((k-i+1) \Delta t)\right]\right\}
\end{gathered}
$$

Therefore, for impact force solved:

$$
\begin{aligned}
& {\left[\frac{F_{k}}{k_{2}}\right]^{\frac{2}{3}}=v_{0}(k \Delta t)-\frac{\Delta t^{2}}{m_{s}} \sum_{i=1}^{k} F_{i}\left(k-i+\frac{1}{2}\right)} \\
& -\sum_{n=1}^{\infty} \sum_{m=1}^{\infty} \frac{\widetilde{W}_{n m} \widetilde{W}_{n m}\left(x_{0}, y_{0}\right)}{I_{0} K_{n m} \omega_{n m}^{2}} \times \frac{1}{\omega_{n m}} \sum_{i=1}^{k} F_{i}\left\{\cos \left[\omega_{n m}((k-i) \Delta t)\right]-\cos \left[\omega_{n m}((k-i+1) \Delta t)\right]\right\}
\end{aligned}
$$

To fined $F_{k}$ in step time $k$, from zero to impact duration. 